limited to the passage of the impulse, but also that the injection of it suddenly into an artery in close relation to the muscle gives rise to a twitch very similar both from mechanical and from electrical records to that produced on nerve stimulation.

F. Buchtal (Copenhagen) has made a more minute study of the structures involved in this transmission. Working with single muscle fibres from the lizard, he measured the resting and active potentials on the surface of the muscle and the nerve-muscle junction. There is a resting potential of some $50 \mathrm{mV}$. between junction and fibre, and this difference is reversibly reduced to zero by the drug curare, which simultanoously produces a reversible nerve-muscle paralysis.

Z. N. Bacq (Liège) treated from a much wider zoological aspect the question of chemical transmission, and showed that, though acetyl-choline may be regarded as transmitter over a great range, yet this substance is not present in the Protochordates, the Crustaicea, or the Colenterates.

J. C. Eccles (Oxford) considered the application to ganglion and central nervous system. He presented evidence for two transmitters, one rapid, and apparently related to the electrical effect, the second slower and probably related to acetyl-choline.

This same duality was advocated by C. F. A. Pantin (Cambridge) in his opening communication. His own work on sea anemones and crabs shows that facilitation at the nerve-muscle junction depends upon both the number and the frequency of impulses arriving there. A chemical transmitter is suggested because the time relations of the decay of facilitation are much longer than those of electrical effects, and are not dependent upon ions as are the electrical phenomena. On the other hand, the accumulation of facilitating chemical is never sufficient to set up repetitive impulses on its own account, nor are the ionic changes which increase facilitation capable of allowing a first impulse to pass; it seems that in these systems it is necessary to have the electrical change associated with the present impulse as well as the chemical facilitation resulting from a previous impulse. He also pointed out that the study of comparative physiology is rich in examples of the same end being achieved by different means, and of the same means being employed to different ends, or to no end at all. The fact that a certain chemical has important function in one tissue and that it is also present in another tissue, cannot be used as indicating that it has any biological significance in the second case.

Prof. E. D. Adrian, starting the open discussion, was also of the opinion that both electrical and chemical conditions are involved in transmission. He pointed out, however, that the secretion of mole. cules so strongly polar as those of acetyl-choline may involve quite considerable potential changes, while on the other hand the diffusibility of acetyl-choline through lipoids makes this ion of importance in the passage of electricity through cell walls. It is therefore possible that some of the phenomena variously considered as electrical or chemical may in fact be merely two aspects of the same process.

W. A. H. Rushtos.

\title{
The Individuality of Gliadin
}

\section{By Prof. A. G. Kuhlmann, Research Institute of the Baking Industry, Moscow}

$\mathrm{T}$ HE principal proteins of wheat are, as is well known, glutenin and gliadin. These proteins, upon the interaction of wheat flour with water, form the so-called gluten, the quality and quantity of which is of such great significance in the baking of bread, in the manufacture of macaroni, noodles and similar products. In the numerous works devoted to the proteins of wheat, a great deal of attention is paid to the question of the individuality of glutenin and gliadin.

Considering gluten to be a natural high polymer, we must come to the conclusion that it represents a complex of proteins, forming micelles of various length. Such a conclusion has been fully confirmed by an investigation which I conducted in my laboratory, which showed as well that the longest and most stable micelles are those of glutenin. Gliadin consists of shorter micelles, which are less stably built and more flocculent than those of glutenin.

According to the literature, the gliadin of wheat is best extracted by a 70 per cent solution of ethyl alcohol. A study of the influence of the concentration of ethyl alcohol on the peptization of the proteins of gluten carried out in my laboratory gave the following picture (Fig. 1). In one series of experiments, samples of one and the same gluten were treated with alcohol-water solutions. The experiments were ropeated, this time a given sample of gluten being treated with alcohol solutions of increasing concentration (alcohol of higher concentration being used only after complete extraction with alcohol of the preceding lower concentration).

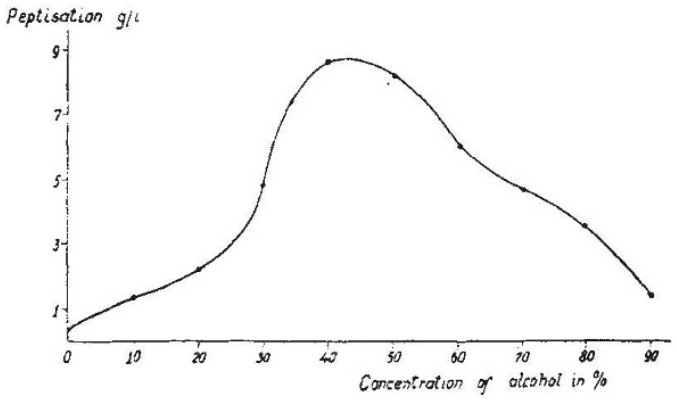

Fig. 1.

Results of these experiments are shown in Fig. 2. The data of both these series of experiments are in good agreement with each other and show that maximum peptization occurs with alcohol having a concentration of about 40 per cent. The 
investigation of several kinds of gluten gave an analogous picture.

When gluten is treated with alcohol of low concentration (up to 30 per cent $\mathrm{C}_{2} \mathrm{H}_{5} \mathrm{OH}$ ) it retains its cohesive properties and changes only slightly in its external appearance. Upon treatment with 40 per cent alcohol, it is greatly altered ; it loses its cohesive properties and begins to disintegrate into separate particles. The supernatant liquid becomes turbid and viscous.

It is a striking fact that peptization of gluten by alcohol of various concentrations falls considerably more sharply after reaching a definite maximum, in the case illustrated by Fig. 2 than that by Fig. 1. This can be accounted for by the presence in gluten of a less highly aggregated and more easily peptizable fraction the solubility of which reaches a maximum with concentrations of alcohol of about 40 per cent. This fraction, through interaction with the more highly aggregated and less soluble fraction of gluten by means of absorption, acts as a peptizer of the latter. It also conditions the stability of the sol obtained. This fraction, when separate samples of gluten are extracted by different concentrations of alcohol (Fig. I), asserts its action even at high

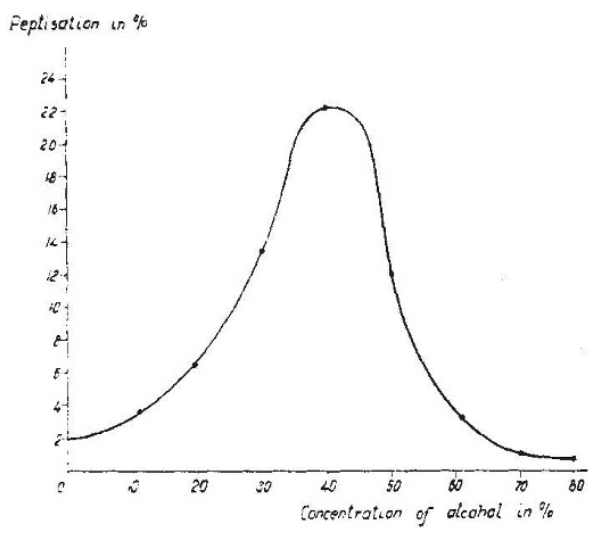

Fig. 2.

concentrations; while when one and the same sample is extracted by alcohol of different concentrations, the alcohol solutions containing more than 40 per cent $\mathrm{C}_{2} \mathrm{H}_{5} \mathrm{OH}$, devoid of this less highly aggregated fraction of gluten due to its solution by the alcohol solutions of lesser concentrations, show a sharp decrease in peptizing properties (Fig. 2).

A study of the peptization of the proteins of gluten as a tricomponent system, sol of gluten - alcohol water, using the method of the triangular diagram of Gibbs, was also carried out in my laboratory on twelve specimens of wheat. In general, they all gave analogous results. A typical example is shown in Fig. $3(a)$. Isopeptides (lines connecting the points of equal quantities of peptized colloids) clearly show a definite maximum of peptization at a definite concentration of alcohol ( $40-50$ per cent). If isoprotein sections (lines I, II, III) parallel to the side $B C$ and connecting points of equal quantities of protein are drawn, then the above-mentioned maximum is seen still more clearly (Fig. $3(b)$ ).

If proteins are extracted with 40 and 70 per cent alcohol-water solutions from separate samples of a given specimen of gluten, then we obtain two products, different from each other with respect to their behaviour towards water (swelling and hydration). Thus the protein fraction extracted with 40 per cent alcohol from the gluten of the wheat Lutescens 0329 imbibes $1.63 \mathrm{gm}$. of water (calculated on the basis of $1 \mathrm{gm}$. of dry substance), while gliadin (that is, the fraction extracted from that same sample of gluten with 70 per cent alcohol) imbibes $1.83 \mathrm{gm}$. The corresponding fractions extracted from the gluten

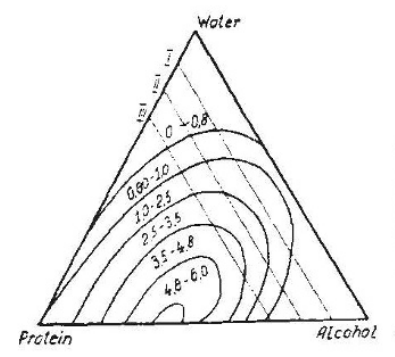

(a)

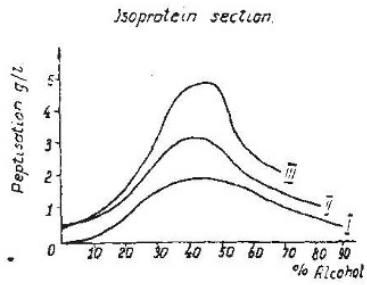

(b)
Fig. 3.

of the wheat Ferrugineum $H 622$ gave a still more distinct picture: here gliadin imbibed $1.08 \mathrm{gm}$. of water while the ' 40 per cent-fraction' imbibed $0.91 \mathrm{gm}$. The gliadin of that wheat increased its volume by 158 per cent on swelling, while the ' 40 per cent-fraction' increased by 135 per cent.

The above data, as well as other observations noted in my laboratory, give me reason to maintain that the gliadin of wheat is not a chemical individual. It represents an adsorption complex of at least two fractions, which I name $\alpha$ - and $\beta$-gliadin. In its properties (swelling, peptization, etc.) $\beta$-gliadin approaches glutenin. B-gliadin dissolves as a result of interaction by means of adsorption with the more easily peptizable fraction, $\alpha$-gliadin, which forms the main mass of Osborne's gliadin.
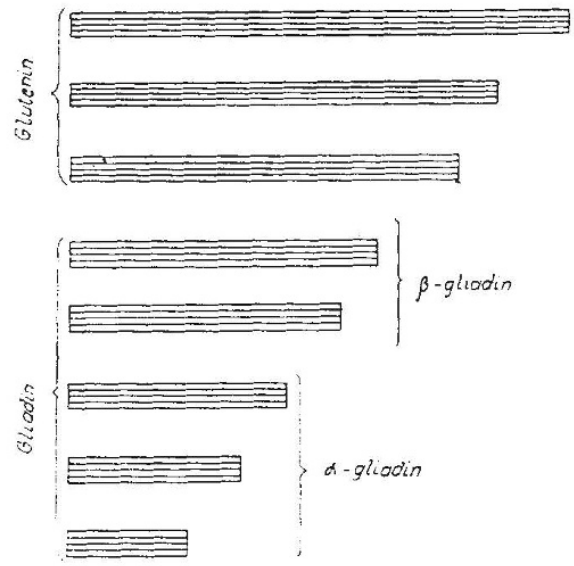

Fig. 4.

In the light of this work, the distribution of proteins of the various fractions of gluten according to the length of their micelles may be represented schematically as in Fig. 4.

Research in this field is being continued. 